www. revistad y o. com

\title{
Casos en formato Cómic para la docencia: Innovando en el Estudio de Casos en Dirección de Operaciones ${ }^{1}$
}

\author{
J.M. Maqueira, J. Moyano-Fuentes, P. Nuñez-Cachoa y D. de Oliveira Diasa
}

Recibido: 13 de Septiembre de 2019

Aceptado: 26 de Noviembre de 2019

https://doi.org/10.37610/dyo.v0i71.575

\section{Resumen}

Este trabajo persigue el objetivo principal de introducir una innovación en la técnica del Estudio de Casos con fines docentes, concretamente un nuevo formato de presentación: el Cómic. A partir de la revisión de la literatura efectuada se pone de manifiesto el potencial de utilizar esta herramienta en la enseñanza universitaria. En el trabajo se expone el proceso seguido en la elaboración del formato propuesto y los resultados obtenidos, mostrando el caso de un tema estratégico de Dirección de Operaciones. Así, se introducen los conceptos que se tratarán en el tema a través del Cómic, se crean los personajes que reflejan los roles típicos de los directivos que intervienen en las decisiones del caso y, por último, se desarrollan una serie de cuestiones relacionadas con dicho caso que deben responder los alumnos de forma oral o escrita, individual o en grupo. Para valorar su utilidad se muestran los resultados alcanzados con la aplicación del caso en formato Cómic, que se presenta en este trabajo como ejemplo, en un grupo de estudiantes de máster y que se contrasta con la opinión de un colectivo a nivel nacional de profesores expertos en el ámbito de Dirección de Operaciones. Los resultados de dos encuestas dirigidas a ambos colectivos muestran la conveniencia de utilizar los casos en formato Cómic como método de enseñanza tremendamente útil en la adquisición de conocimientos y competencias en el ámbito de Dirección de Operaciones.

\section{Palabras clave}

Enseñanza; Investigación; Estudio de Casos; Innovación en Estudio de Casos; Cómic.

\section{Introducción}

Actualmente el proceso de enseñanza/aprendizaje está centrado en el alumno, quien adquiere un papel protagonista en la asimilación de contenidos y en la adquisición de competencias. En este sentido, en las asignaturas de Organización de Empresas, se emplea con frecuencia la técnica del Estudio de Casos como herramienta de enseñanza-aprendizaje de los contenidos teóricos impartidos. El Estudio de Casos es un método de investigación cualitativa que se ha utilizado

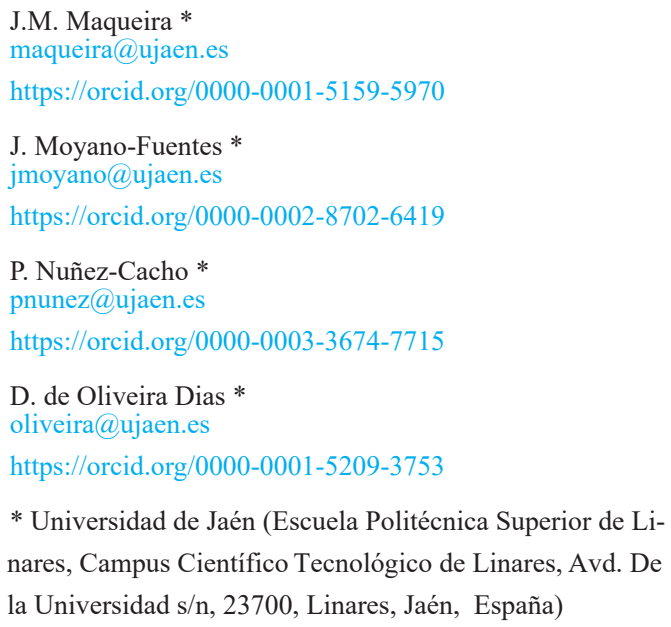

ampliamente para comprender en profundidad la realidad (Eisenhardt, 1989; Yin, 1994). La particularidad más característica de ese método es el estudio intensivo y profundo de un/os caso/s que muestran una situación con cierta intensidad (Yin, 1994). El Estudio de Casos es una técnica bastante desarrollada y utilizada en las Ciencias Sociales (Eisenhardt, 1989; Yin, 1994). Tradicionalmente, en la aplicación de esta técnica a la docencia, se expone una situación concreta o caso mediante texto, y el alumno debe leer dicho texto con atención y asimilarlo, extrayendo la información contenida en el caso, identifica do los conceptos teóricos a los que hace referencia y respondiendo a unas preguntas concretas que se le realizan sobre el contenido del texto leído.

La innovación en esta técnica se está produciendo en los formatos de presentación de los casos. Nuevos formatos persiguen llamar la atención del alumno y aumentar su interés, y se ha ensayado con éxito la utilización de formatos de vídeo para presentar los casos (Vídeo-Casos), por ejemplo, con vídeos disponibles en YouTube (Kender et al., 2015; Figueiredo et al., 2014). Este tipo de formato introduce aspectos y connotaciones lúdicas típicas de técnicas como la gamificación (Arias, Bustinza y Djundubaev, 2016) y que podrían aumentar el interés del alumno por el caso expuesto y por la resolución de las cuestiones planteadas.

\footnotetext{
${ }^{1}$ Una versión previa de este trabajo se presentó en el XXVII Congreso Universitario de Innovación Educativa en las Enseñanzas Técnicas (XXVII CUIEET).
} 
Precisamente, en esta misma línea, en este trabajo perseguimos el objetivo de explorar una posibilidad de innovación, que es la presentación del caso de estudio mediante formato Cómic, así como la investigación de las percepciones de alumnos y profesores sobre la utilidad de dicho formato. Un Cómic puede ser definido como una estructura narrativa formada por la secuencia progresiva de pictogramas, en los cuales pueden integrarse elementos de escritura fonética (Arango, Gómez y Gómez, 2009).

Para conseguir el objetivo propuesto, el trabajo ha sido estructurado como se describe a continuación. Tras esta introducción se muestra el marco teórico, o trabajos de la literatura previa relacionados. A continuación se muestra la metodología utilizada. Posteriormente se recogen los resultados obtenidos. Finalmente, se muestran las principales conclusiones del trabajo.

\section{Marco Teórico}

El Estudio de Casos fue popularizado por la Harvard Business School para su uso en cursos sobre negocios (Christensen, 1989). El método consiste en captar una parte de la realidad y dar una descripción detallada de un ejemplo concreto, para así hacer posible una discusión sobre temas claves. En la literatura son muchos los trabajos que destacan la importancia de utilizar el Estudio de Casos en el proceso de enseñanza/aprendizaje de contenidos sobre gestión de empresas (por ejemplo, Parast, 2010; Saladin y Shafer, 2006; Hammond, 2002; Ress y Porter, 2002).

En el Estudio de Casos, a través de la lectura del mismo, los estudiantes pueden analizar, entender y capturar la complejidad de una situación real de negocios, adoptando el punto de vista de una persona que toma decisiones y relacionando la teoría con la práctica (Desiraju y Gopinath, 2001). Sin embargo, presentar una situación real de negocios no es una tarea fácil, ya que la complejidad de la práctica gerencial es muy amplia y es difícil reducirla con fines didácticos (Štrach, Boleslav y Everett, 2008) y en este sentido hay críticas al método que cuestionan su eficiencia (Shugan, 2006). No obstante, también existen investigaciones que avalan su uso. En una investigación sobre la utilización del Estudio de Casos para el aprendizaje en el área de Dirección de Operaciones (Parast, 2010), se ha encontrado que el Estudio de Casos puede mejorar los resultados de aprendizaje de los estudiantes, principalmente en el desarrollo de habilidades como la toma de decisiones y la resolución de problemas, así como de la comprensión y aplicación de los conceptos teóricos, además de mejorar la autoconfianza de los estudiantes. Por lo tanto, el autor concluye que es recomendable que el Estudio de Casos sea parte integrante de cursos de Dirección de Operaciones (Parast, 2010).

Por otra parte, las experiencias previas de utilización de los Cómics con fines docentes son muy escasas (Jamal, Ibrahim y Surif, 2019). Y los escasos trabajos existentes (Demirci y Ozyurek, 2017; Yin y Fitzgerald, 2017; Balim, Inel y Ozcan, 2016; Fatimah y Widiyatmoko 2015; Balim et al., 2014; Balim et al., 2013; Inel y Balim, 2013; Murtiningrum, Ashadi y Mulyani, 2013; Balim et al., 2012; Inel y Balim, 2011; Birisci y Metin, 2010) han sido aplicados en enseñanzas básicas y medias, siendo prácticamente inexistente su aplicación en la educación superior universitaria (Jamal, Ibrahim y Surif, 2019).

Investigaciones sobre estas experiencias previas (Jamal, Ibrahim y Surif, 2019) han encontrado que este tipo de formato fomenta el pensamiento creativo, el pensamiento abstracto y el pensamiento crítico del alumno, (Demirci y Ozyu-rek, 2017; Fatimah y Widiyatmoko, 2015; Yin y Fitzgerald, 2017) y mejora la motivación y el interés de aprendizaje (Balim et al., 2012; Inel y Balim, 2011). Y el desarrollo de estas capacidades de los alumnos es de gran interés en la educación superior en general, y en las enseñanzas técnicas universitarias en particular. Además los Cómics ayudan a los estudiantes a experimentar con métodos de aprendizaje diferentes al de la lectura tradicional, pudiendo analizar un tema determinado desde diferentes perspectivas, lo que permite a los estudiantes profundizar en los conceptos que subyacen en los temas estudiados (Balim et al., 2013; Balim, Inel y Ozcan, 2016; Birisci, Metin y Karakas, 2010; Murtiningrum, Ashadi y Mulyani, 2013). En esta línea, Balim et al. (2014) al examinar la opinión de profesores sobre la utilización del aprendizaje basado en problemas a través de Cómics encontraron, tras su uso experimental, una valoración muy positiva sobre su empleo. Con respecto a la opinión de los estudiantes, se concluye que el método ofrece la oportunidad de aprender divirtiéndose y de participar activamente en las clases (Inel y Balim, 2013).

Los argumentos anteriores justifican que es pertinente aplicar el Cómic con fines de enseñanza/aprendizaje a la educación superior en general y a la Dirección de Operaciones en particular, y no hay razones para limitarlo sólo a la educación elemental, ya que el Cómic es un medio muy popular y presenta gran potencial en personas de todas las edades (Arango, Gómez y Gómez, 2009).

En la revisión realizada de la literatura existente sobre la utilización de los Cómics con fines docentes (Jamal, Ibrahim y Surif, 2019) no hemos encontrado antecedente alguno sobre la utilización del formato Cómic como parte de la técnica del Estudio de Casos aplicada a la docencia, aunque sí antecedentes que proponen la utilización de este formato como elemento de mediación para la enseñanza en la educación superior. En este sentido, Arango, Gómez y Gómez (2009) investigan este posible uso. Mediante una muestra de informantes clave y por medio de observaciones, entrevistas en profundidad, redacción de diarios de campo $\mathrm{y}$ estudio de fuentes documentales, sus principales conclusiones son que el Cómic tiene potencial didáctico, que ha sido poco utilizado para enseñar, y que las instituciones de educación 
superior deben propiciar su uso e investigar para validar este potencial (Arango, Gómez y Gómez, 2009). Precisamente, para cubrir este gap, en este trabajo se presenta el desarrollo de una serie de Estudio de Casos en formato Cómic (Comic-Cases) para ser aplicados en la docencia Universitaria sobre Dirección de Operaciones. Para la elaboración de estos Cómics se ha seguido un proceso similar al utilizado en la elaboración de otras obras de narración visual como pueden ser las series televisivas o las películas. Este proceso nos ha permitido elaborar los Comic-Cases para este fin

\section{Metodología}

El proyecto ha sido llevado a cabo por un equipo multidisciplinar formado por tres profesores del área de Organización de Empresas, junto con un colaborador externo que realiza la ilustración gráfica de los Cómics.

El proceso de creación de los Comic-Cases se inicia con una reunión inicial o Kick-off del proyecto. En esa reunión, mediante un brainstorming se establecen los requisitos que regirán la elaboración de los Cómics. Así, se establece el ámbito sobre el que girarán los Comic-Cases, que se centrarán en las decisiones estratégicas de Dirección de Operaciones. En concreto se identifican seis grandes decisiones sobre las que se articularán los Cómics: (1) decisión sobre el producto; (2) el proceso; (3) la localización y capacidad; (4) las Tecnologías de la Información y la Innovación; (5) la gestión de cadena de suministro y (6) la aplicación de Lean Management. También se acuerda establecer un "hilo conductor" bajo el que se articulan todos los casos. Como hilo conductor, buscando que la novedad y atractivo del caso perdure lo más posible en el tiempo, se propone emular el caso de Tesla Motors, el popular fabricante de vehículos eléctricos, creando una empresa ficticia (denominada Testa Motors) y personajes ficticios inspirados en su trayectoria con respecto a las decisiones estratégicas de Dirección de Operaciones que ha aplicado. En este sentido, se pensó en indicar de forma expresa en los Cómics que la empresa y personajes reflejados en el caso son ficticios e ilustran decisiones estratégicas en Dirección de Operaciones con fines docentes, sin vinculación con la realidad más allá de la pura coincidencia. También se decidió la longitud de 6 páginas impresas (para que se pueda trabajar con él en clases de una hora de duración), más una página a modo de portada y otra final con las cuestiones sobre el caso (8 páginas en total).
En una segunda reunión se identifican y caracterizan los personajes principales, que aparecerán en los distintos casos y se asigna a cada miembro del equipo investigador la tarea de realizar dos guiones de los casos inicialmente identific dos. La tarea de realización del guión presenta a su vez dos distintas fases: (a) documentación bibliográfica, utilizando para ello como referente un libro muy actual sobre Dirección de Operaciones, decisiones estratégicas (Arias-Aranda y Minguela-Rata, 2018), así como otros más tradicionales (Heizer y Render, 2011; Dominguez-Machuca et al., 1995) y (b) elaboración de un guión corto, que se desarrolla en la empresa ficticia utilizando los personajes creados, y que debe recoger e ilustrar los principales aspectos teóricos asociados a la decisión en cuestión.

En la siguiente etapa del proceso, el coordinador del proyecto recibe, verifica y adapta el guión que debe dar lugar a un Cómic de 6 páginas. En esta etapa, es necesario utilizar el pensamiento visual y la narración visual de historias (Osterwalder y Pigneur, 2011) imaginando escenas y tramas, así como la capacidad de síntesis que permita mostrar mucho contenido en diálogos cortos y cuadros de texto de reducidas dimensiones. En esta fase, material gráfico muy técnico y que complementa al contenido en texto es también realizado por el coordinador del proyecto. Se trata de una etapa crítica, pues condiciona las siguientes.

A continuación, el guión es enviado al diseñador gráfico externo que lo transforma en viñetas con ilustraciones y textos, bien como diálogos entre personajes o como cajas explicativas. El coordinador recibe el trabajo del colaborador externo, y verifica el resultado en todas sus facetas (dibujos y texto), proponiendo las modificaciones oportunas. Las modificaciones se proponen al diseñador gráfico, que las implementa y devuelve el nuevo resultado. Esto se realiza en un proceso cíclico, hasta obtener el resultado final deseado (en general tres iteraciones fueron suficientes).

Acto seguido, para cada uno de los Comic-Cases elaborados, el profesor-guionista elabora una serie de cuestiones que deberán ser resueltas por el alumno, y que se incorporan en la última página del Cómic. Se ha optado por un número amplio de cuestiones (unas 25 por cada caso) con el objetivo de que cada profesor utilice luego las que estime oportunas.

En una última reunión se analiza y acepta el resultado final por todos los integrantes del proyecto. Este proceso, que se muestra en la siguiente figura (Figura 1), ha permitido la elaboración hasta el momento de 4 Cómics, y otros dos se encuentran en avanzado estado de desarrollo. 
Figura 1 Diagrama del proceso.

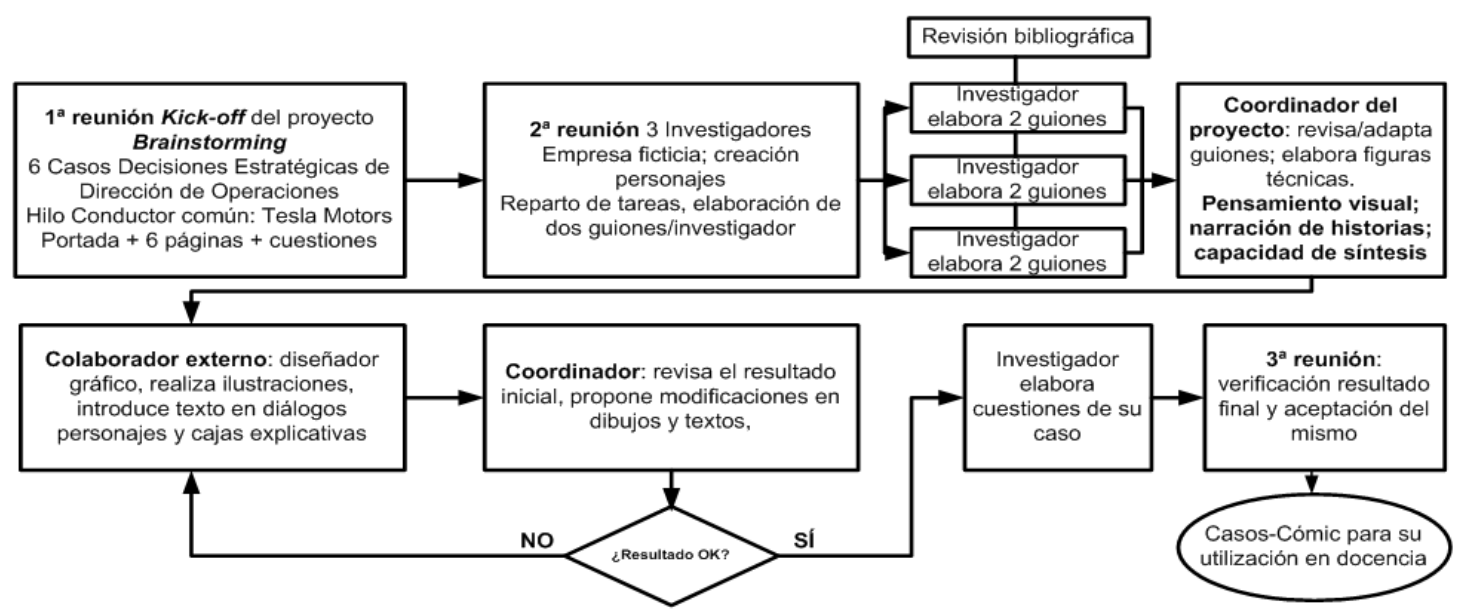

El proyecto, también presenta una vertiente investigadora que permitirá verificar la efectividad y utilidad de los casos. Los resultados obtenidos (Comic-Cases), están siendo utilizados de forma incipiente en la docencia de Dirección de Operaciones, en asignaturas de grado y Máster del área de Organización de Empresas, en la Escuela Politécnica Superior de Linares (Universidad de Jaén). Se elaboró una encuesta para su aplicación a los alumnos, con el fin de recoger su opinión sobre la utilidad del Cómic en el aprendizaje de las asignaturas. Para validar la utilidad del formato se diseñó también una encuesta para recoger la opinión de los profesores de Dirección de Operaciones. Conocer la opinión de alumnos y profesores, permitirá, en el futuro próximo, establecer recomendaciones y buenas prácticas para la aplicación de la innovación de Casos en formato Cómic en la docencia de Dirección de Operaciones.

\section{Resultados}

\subsection{Los Casos en formato Cómic}

El proceso identificado y descrito en el apartado anterior, ha permitido obtener hasta el momento, en primer lugar, material didáctico en forma de cuatro casos sobre decisiones estratégicas de Dirección de Operaciones en formato Cómic. Además, otros dos casos, si bien no están totalmente concluidos, se encuentran en avanzado estado de desarrollo. La Figura 2 muestra a modo de ejemplo uno de los resultados obtenidos.

En el ejemplo de la Figura 2 se desarrollan cuestiones específicas sobre la decisión estratégica de Dirección de Operaciones de selección y diseño del producto. En este Cómic en concreto se introducen conceptos tales como: Decisiones sobre atributos del producto (calidad, diseño y estilo); Ciclo de vida del producto; fases del proceso de creación de nuevos productos (generación de ideas, selección de ideas, análisis de viabilidad, desarrollo del producto, prueba de mercado, comercialización); Diseño para la fabricación; Ingeniería de valor; Ventajas del primer movedor; Innovaciones incrementales y radicales; Decisión sobre producir o comprar; Previsión de la habitual en el método del Estudio del Caso, en la información que se entrega al alumno se utilizan, aplicados de forma práctica, conceptos que previamente se han visto en la teoría. En el caso en formato Cómic mencionado anteriormente, se utiliza como soporte bibliográfico teórico el libro Arias-Aranda y Minguela-Rata (2018). Además se introducen datos que permiten apoyar esos conceptos, por ejemplo identificando las fases del proceso del diseño de nuevos productos o del ciclo del vida del producto. demanda; Alianzas estratégicas; entre otros. Como es 
Figura 2 Ejemplo de resultado: Comic-Case Decisión Estratégica sobre selección y diseño del producto.
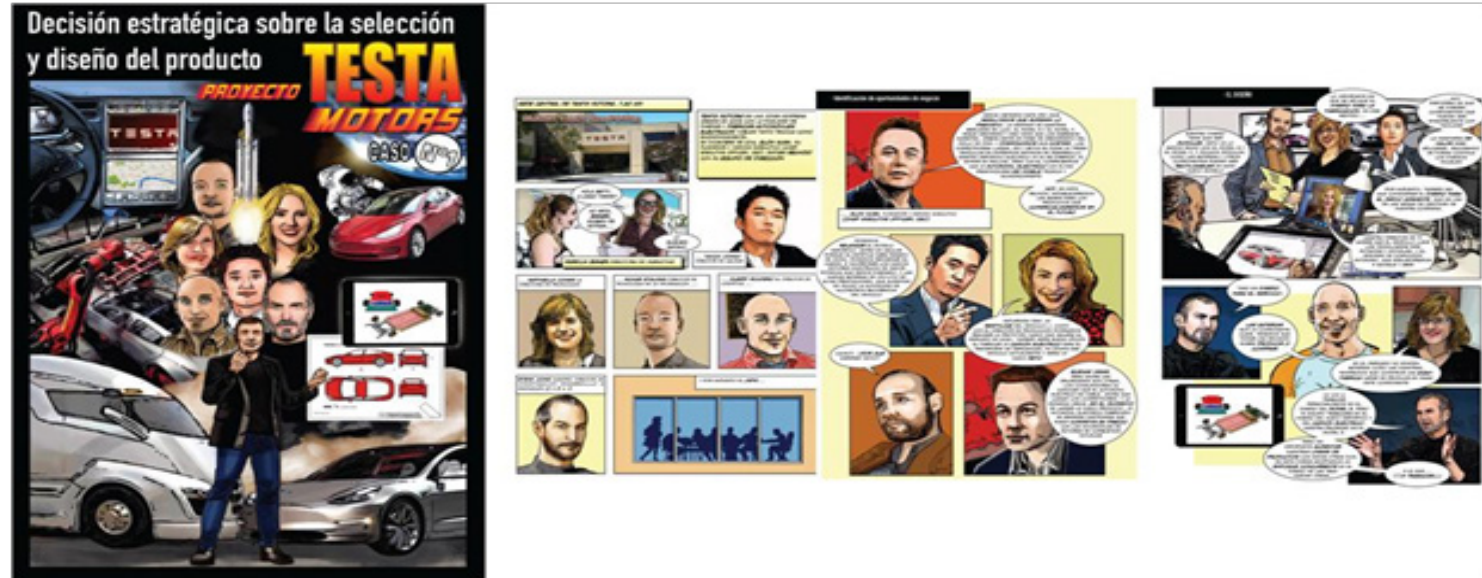

Para la realización de los Cómics se han creado personajes específicos que aparecen en los distintos casos (ver Figura 3). Estos personajes reflejan roles típicos de dirección relacionados con la Dirección de Operaciones, como son el de Dirección General o CEO, Dirección de Logística o Cadena de Suministro, Dirección de Producción, Dirección de Marketing, Dirección de Calidad y Dirección de Tecnologías de la Información. Estos roles están involucrados en las decisiones que una empresa toma en relación con las operaciones y por ese motivo existen personajes específicos que reflejan estos roles. Los Cómics realizados se estructuran en secciones que se distinguen mediante un rótulo indicativo insertado en determinadas páginas. Así, por ejemplo, en el Cómic sobre la Decisión estratégica de selección y diseño del producto, las secciones se corresponden con las distintas fases existentes en el desarrollo de un nuevo producto (ver Figura 3).

Figura 3 Comic-Case

Decisión Estratégica sobre selección y diseño del producto.

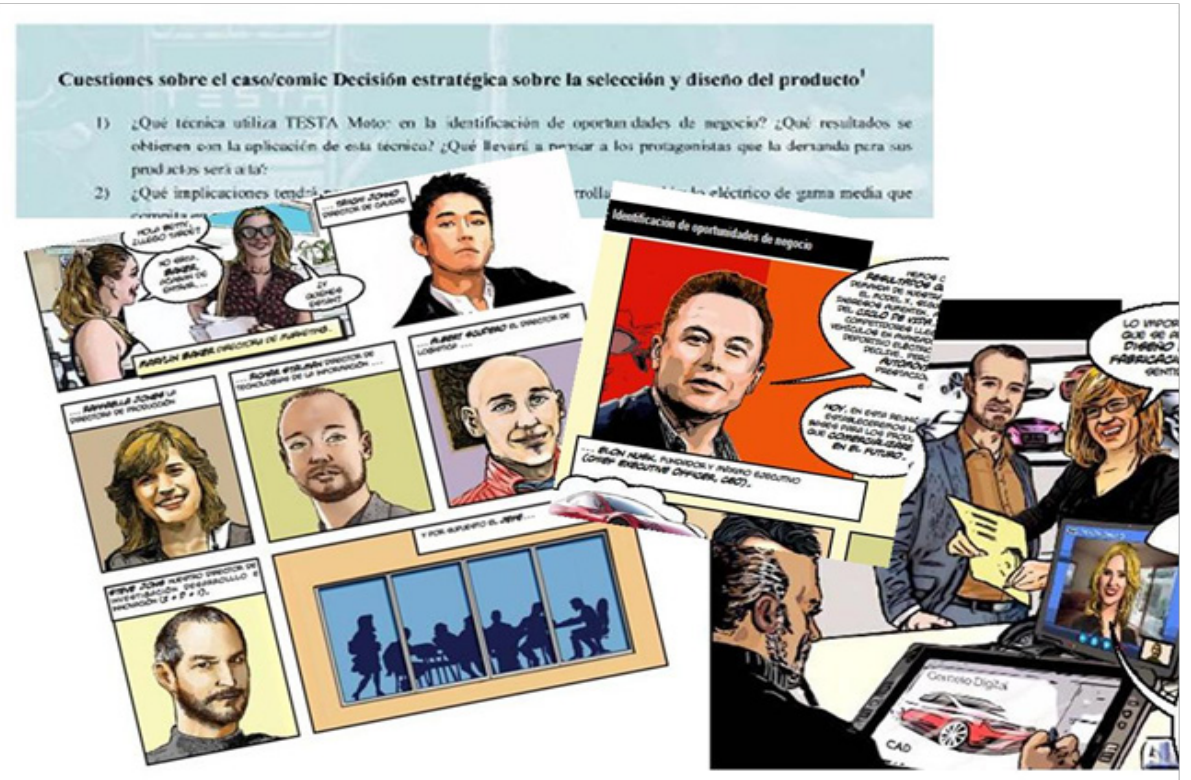


Al final de cada Comic-Case se encuentran las cuestiones relacionadas con el caso expuesto (ver Figura 3), de acuerdo con los contenidos teóricos trabajados en clase con anterioridad. Cada Comic-Case cuenta con aproximadamente 25 cuestiones.

\subsection{La experiencia de aplicación}

También se han obtenido resultados de la experiencia de aplicación incipiente de dicho material en el proceso de enseñanza-aprendizaje. El Comic-Case sobre la decisión estratégica de Dirección de Operaciones "Selección y diseño del producto" presentado en el apartado anterior fue utilizado en una asignatura del Máster en Industria 4.0 de la Universidad Jaén. El grupo al que se aplicó era un grupo reducido de 16 alumnos, en el que dominaban los alumnos con experiencia profesional o que trabajaban en empresas. Con un rango de edad comprendido entre los 23 años y los 50 años. En la dinámica de clase, los alumnos trabajaron de forma individual con Estudios de Casos en su formato tradicional (formato texto). Posteriormente se utilizó el Estudio de Casos en formato Cómic. Se le entregó a cada alumno un ejemplar impreso a color del Cómic presentado en la sección anterior. También de forma individual, los alumnos trabajaron con este formato en clase, y respondieron de forma individual y escrita a diversas cuestiones planteadas. Aunque el caso en formato texto y en formato Cómic presentaban situaciones concretas distintas, el contenido teórico que subyacía en ambos era similar (selección y diseño del producto). Al final de la asignatura los estudiantes fueron invitados a responder a una encuesta elaborada para obtener su percepción. La encuesta presentaba una primera parte de captura de datos demográficos de los encuestados (edad, formación, sexo, situación laboral). Una segunda parte captura su impresión sobre la utilidad del Cómic para el desarrollo de determinadas capacidades del alumno. Una tercera parte hace una comparativa entre el método en formato Cómic y el método en formato texto, de tal forma que los alumnos deben indicar qué formato le parece más adecuado para conseguir determinados resultados. La encuesta finaliza con dos preguntas que capturan la satisfacción del alumno con el formato texto o con el formato Cómic y si recomendarían más un formato que el otro (Figura 4).

Fueron obtenidas 15 respuestas válidas de estudiantes. La muestra estaba compuesta principalmente por alumnos entre 31 y 35 años $(33,33 \%)$ y entre 46 y 50 años $(26,67 \%)$, hombres $(80 \%)$ y que estaban trabajando $(80 \%)$. Entre los resultados se destaca que el $93 \%$ de los estudiantes demuestra estar más satisfecho con el Estudio de Casos en formato Cómic que en formato texto (De acuerdo 46,67\% y Totalmente de acuerdo 46,67\%) (ver Figura 4). Además el $100 \%$ de los estudiantes afirmaron que recomendarían más la utilización del método en formato Cómic que el método en formato texto (De acuerdo $40 \%$ y Totalmente de acuerdo $60 \%$ ) (ver Figura 4).

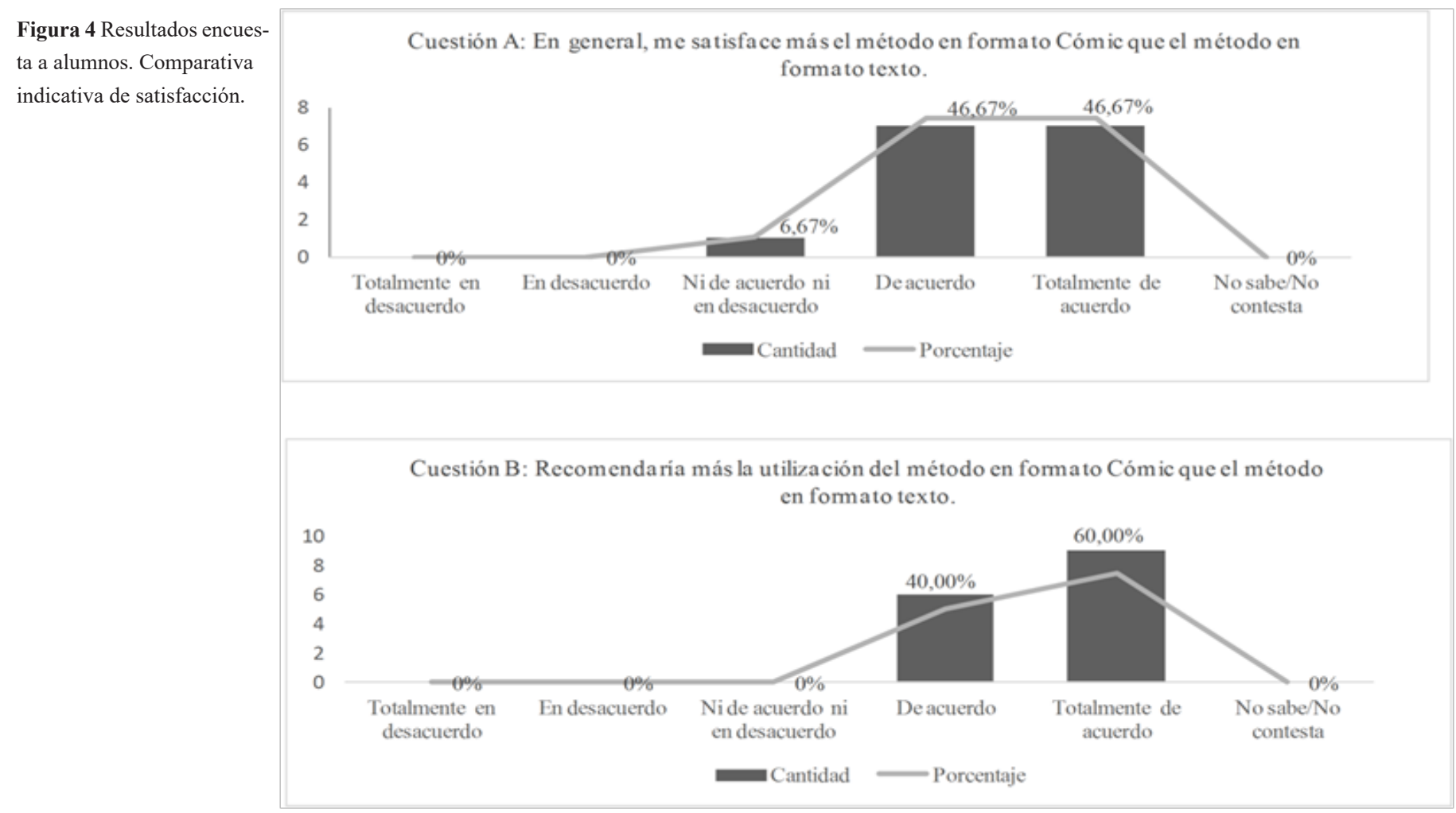


Por otro lado, también fueron obtenidas respuestas de profesores sobre la utilización del Estudio de Casos en formato Cómic. Durante el OMTech 2019, workshop sobre innovación docente en Dirección de Operaciones organizado por la Asociación Científica de Economía y Dirección de Empresas (ACEDEDOT), que acontece de forma anual y reúne investigadores y profesores de Dirección de Operaciones y Tecnología de las principales universidades españolas, fue presentado uno de los casos en formato Cómic a los profesores participantes. En el evento cada profesor recibió una copia del caso junto con una carpeta con una dirección web y un código en formato Quick Response (QR) para acceder a una encuesta en formato online. La encuesta fue elaborada con tres partes principales, de forma similar a la encuesta aplicada a los estudiantes. La web estuvo activa durante un mes y los profesores se llevaron el material entregado, para que así pudiesen responder a la encuesta después de examinar el material con profundidad. Fueron obtenidas un total de 16 respuestas de profesores con edades prin- cipalmente entre 46 y 50 años $(41,18 \%)$ y entre 51 y 55 años $(29 \%)$, la mayoría hombres $(76 \%)$, profesores titulares de universi-dad $(35,29 \%)$ o ayudantes doctores $(23,53 \%)$. Además, to-dos los encuestados eran de universidades públicas $(100 \%)$ y la mayoría $(76 \%)$ estaba participando en el momento de la encuesta en algún proyecto de innovación docente. En-tre los resultados de la encuesta destacamos que el $75 \%$ de los profesores indicó estar más satisfecho con el Estudio de Casos en formato Cómic que en formato texto (De acuerdo 37,5\% y Totalmente de acuerdo 37,5\%) (ver Figura 5). Como respuesta a la cuestión sobre si se recomendaría más la utilización del método en formato Cómic que el método en formato texto el $37,50 \%$ de los profesores afirmó estar de acuerdo y el 31,25\% afirmó estar totalmente de acuerdo (ver Figura 5). Además, algunos de los profesores consultados sugirieron que los Cómics sean traducidos al inglés, posibilitando su aplicación en asignaturas impartidas en este idioma y pudiéndose expandir su uso en nivel internacional.
Figura 5 Resultados encuesta a profesores. Comparativa indicativa de satisfacción. .

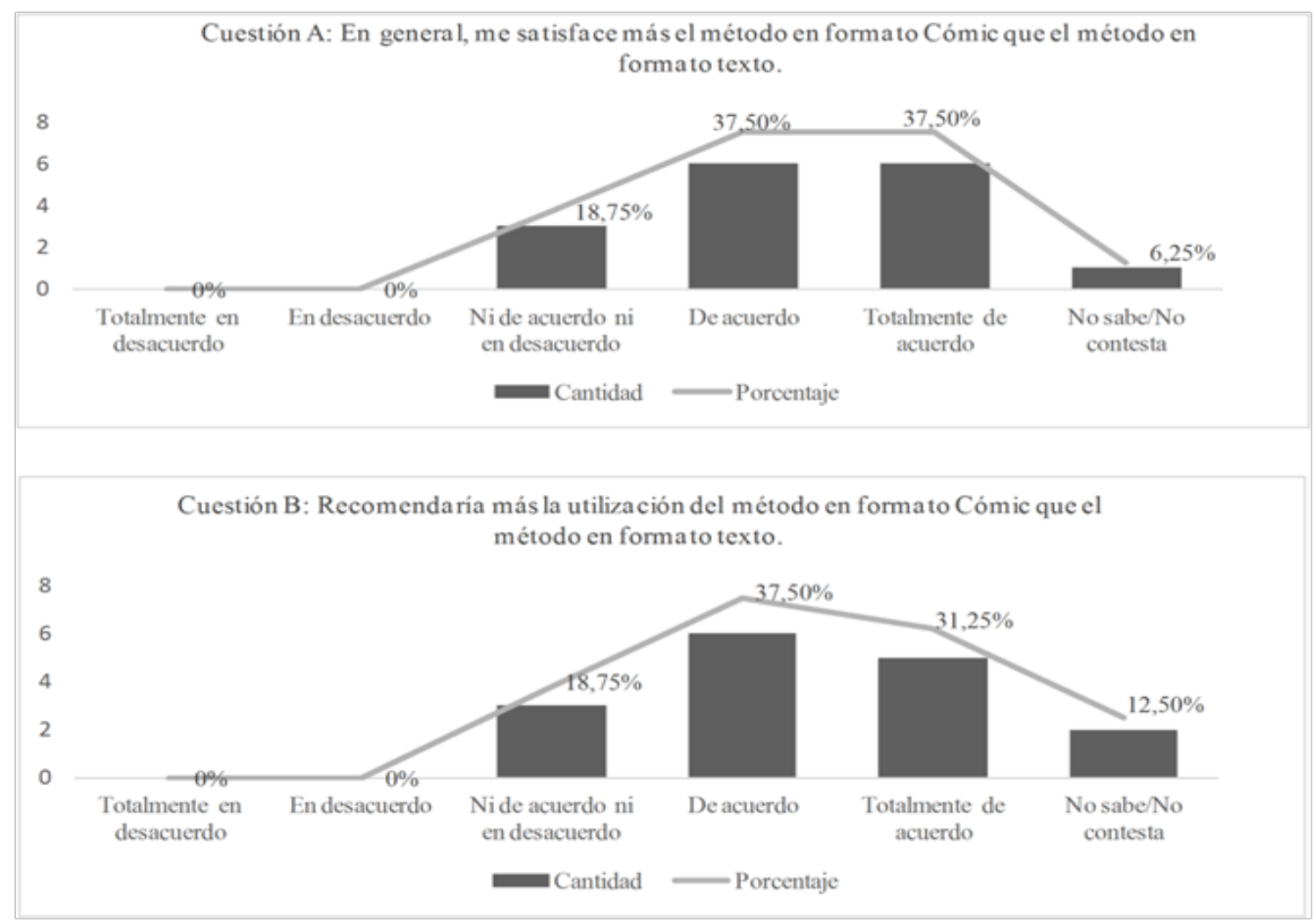


De acuerdo con los datos mostrados con anterioridad, en general los resultados de las encuestas demuestran una alta satisfacción tanto de los alumnos como de los profesores con el Estudio de Casos en formato Cómic.

Con respecto a los resultados académicos de los alumnos, la resolución del caso en formato Cómic obtuvo, de media, una mejor califi ación que la resolución del caso en formato texto (nota media del caso en formato Cómic de 8,5 frente a nota media del caso en formato texto de 7,6 ).

\section{Conclusiones}

Este trabajo presenta el proceso seguido en un Proyecto de Innovación Docente que ha permitido el desarrollo de material didáctico de Estudios de Casos que presentan la información en formato Cómic. El trabajo también muestra los resultados obtenidos, en forma de un Cómic a modo de ejemplo y su descripción, y de la experiencia de su aplicación incipiente al proceso de enseñanza-aprendizaje en la docencia.

Los resultados de las encuestas realizadas sobre la experiencia de aplicación incipiente realizada demuestran que existe un alto nivel de satisfacción con el Estudio de Casos en formato Cómic. En este sentido, tanto los estudiantes como los profesores consultados recomiendan su utilización $\mathrm{y}$, cuando se comparan ambos métodos, señalan una satisfacción superior con el caso mostrado en formato Cómic que con el caso en formato texto.

Con la experiencia incipiente de aplicación en una asignatura de Máster se ha constatado que el Estudio de Casos en formato Cómic es una alternativa válida para facilitar el proceso de enseñanza/aprendizaje en estudios de posgrado. Debido a esto, y a que los estudiantes de grado son generalmente más jóvenes y con características cognitivas que favorecen una mayor atracción hacia el uso de nuevas técnicas de enseñanza/aprendizaje, parece lógico pensar que su utilización podría ser aún más favorable al extenderse a la educación superior en general.

En el futuro, este método de enseñanza/aprendizaje que en este trabajo mostramos, debería explorarse con mayor profundidad de diferentes maneras $y$ en diferentes asignaturas relacionadas con la Dirección de Operaciones. De este modo, a partir de más experiencias de aplicación y obteniendo datos en una muestra mayor de alumnos y profesores sobre la efectividad y utilidad del caso en formato Cómic, sería posible establecer recomendaciones y buenas prácticas de uso. Además, dada la novedad del proyecto, los casos elaborados podrían ser objeto de su publicación en una editorial de prestigio, consiguiendo una gran aplicabilidad potencial de los resultados alcanzados, tanto a nivel de la propia Universidad de Jaén, como de otras universidades de España o Latinoamérica. Otra posibilidad para ampliar la aplicabilidad del método sería la traducción de los Comic-Cases al inglés, lo que posibilitaría su uso a nivel internacional.

\section{Agradecimientos}

Los autores agradecen la financiación recibida de la Universidad de Jaén, mediante el Proyecto de Innovación Docente PID-43_201718 (2017/2019).

\section{Referencias}

ARANGO, J.A., GÓMEZ L.E., y GÓMEZ, M.M. (2009). «El cómic es cosa seria. El cómic como mediación para la enseñanza en la educación superior». Anagramas, 7(14), pp. 13-32.

ARIAS, D., BUStINZA, O.F., y DJUNDUBAEV, R. (2016). «Efectos de los juegos de simulación de empresas y Gamification en la actitud emprendedora en enseñanzas medias», Revista de Educación, 371, pp. 133-156.

ARIAS-ARANDA, D., y MINGUELA-RATA, B. (2018). Dirección de la producción y operaciones. Decisiones estratégicas. Madrid: Editorial Pirámide.

BALIM, A.G., CELIKER, H.D, KACAR, S., EVREVKLI, E., TURKOGUZ, S., INEL; OZCAN E., y ORMANCI U. (2012). «Concept cartoons integrated problem-based learning in science and technology education: An activity sample dance of the heated particules». Western Anatolia Journal of Education Science, 3(5), pp. 6887.

BALIM, A.G., CELIKER, H.D., TURKOGUZ, S., y KACAR, S. (2013). «Concept cartoon samples integrated to PBL scenario towards matter and heat unit». Mediterranean Journal of Educational Research, 14a, pp. 865873.

BALIM, A.G., INEL, D., y OZCAN, E. (2016). «Concept cartoons supported problem based learning method in middle school science classrooms». Journal of Education and Learning, 5(2), pp. 272-284.

BALIM, A.G., TURKOGUZ, A., ORMANCI, U., KACAR, S., EVREKLI, E., y OZCAN, E. (2014). «Teachers' views about problem-based learning through concept cartoons». Journal of Baltic Science Education, 13(4), pp. $458-468$.

BIRISCI, S., y METIN, M. (2010). «Developing an instructional material using a concept cartoon adapted to the $5 \mathrm{E}$ model: A sample of teaching erosion». Asia-Forum on Science Learning and Teaching, 11(1), pp. 116. 
CHRIStENSEN, C.R. (1989). Teaching and the case method. Cambridge, MA: Harvard Business School.

DEMIRCI, F., y OZYUREK, C. (2017). «The effects of using concept cartoons in astronomy subjects on critical thinking skils among seventh grade student». International Electronic Journal of Elementary education, 10(2), pp. 243-254.

DESIRAJU, R., y GOPINATH C. (2001). «Encouraging participation in case discussions: A comparison of the MICA and the Harvard case methods». Journal of Management Education, 25(4), pp. 394-408.

DOMINGUEZ-MACHUCA, J.A., ÁLVAREZ-GIL, M.J., GARCÍA-GONZALEZ, S., DOMINGUEZ-MACHUCA, M.A., y RUÍZ-JIMÉNEZ, A. (1995). Dirección de operaciones. Aspectos estratégicos en la producción y los servicios. Madrid: Mc Graw Hill.

INEL, D., y BALIM, A.G. (2013). «Concept cartoons assisted problem based learning method in science and technology teaching and students' views». Procedia-Social and Behavioral Sciences, 93, pp. 376-380

INEL, D., y BALIM, A.G. (2011). «The effect of concept cartoons assisted problem based learning method on motivation about science learning of secondary students». Usak Universitesi Sosyal Bilimler Dergisi, 2(1), pp. 169188.

EISENHARDT, K.M. (1989). «Building theories from case study research». Academy of Management Review, 14(4), pp. 532-550.

FATIMAH, F., y WIDIYATMOKO, A. (2015). «Pengembangan science comic berbasis problem-based learning sebagai media pembelajaran pada tema bunyi dan pendengaran untuk siswa smp». Unnes Science Education Journal, 4(1), pp. 700-710.

FIGUEIREDO, F., ALMEIDA, J.M., BENEVENUTO, F., y GUMMADI K.P. (2014). «Does Content Determine Information Popularity in Social Media? A Case Study of YouTube Videos' Content and their Popularity». Conference CHI'14, April 26-May 1, 2014, Toronto, Canada.

HAMMOND, J.S. (2002). «Learning by the Case Method». Harvard Business School, 9-376-241.

HEIZER, J., y RENDER, B. (2001). Dirección de la producción. Decisiones estratégicas. Madrid: Prentice Hall.
JAMAL, S.N.B., IBRAHIM, N.H.B., y SURIF, J.B. (2019). «Concept cartoon in problem-based learning: A systematic literature review analysis». Journal of Technology and Science Education, 9(1), pp. 51-58.

KENDER, J.R., HILL, M.L., NATSEV, A., SMITH, J.R., y XIE, L. (2015). «Video Genetics: A Case Study from YouTube». Conference MM'10, October 25-29, 2010, Firenze, Italy.

MURTININGRUM, T., ASHADI A.T., y MULYANI, S. (2013). «Pembelajaran kimia dengan problem solving menggunakan media e-learning dan komik ditinjau dari kemampuan berfikir abstrak dan kreativitas siswa». Jurnal Inkuiri, 2(3), pp. 288-301.

OStERWALDER, A., y PIGNEUR, Y. (2011). Generación de modelos de negocio. Barcelona: Editorial Deusto.

PARASt, M.M. (2010). «Effecti eness of Case Study in Enhancing Student Learning in Operations Management». Operations \& Supply Chain Management, 3(1), pp. 4958.

RESS, D.W., y PORTER, C. (2002). «The use of case studies in management training and development. Part $1 »$. Industrial and Commercial Training, 34(1), pp. 5-8.

SALADIN, B., y SHAFER, S. (2006). «Turning the tables on student analysis assignments». Decision Sciences Journal of Innovative Education, 4(1), pp. 169-173.

SHUGAN, S.M. (2006). «Save research - abandon the case method of teaching». Marketing Science, 25(2), pp. $109-115$.

ŠTRACH, P., BOLESLAV, M., y EVERETT, A.M. (2008). «Transforming research case studies into teaching cases». Qualitative Research in Organizations and Management, 3(3), pp. 199-214.

YIN, R.K. (1994). Case study research. Design and methods, Londres: SAGE Publications Inc.

YIN, Y.K., y FITZGERALD, R. (2017). «Peer learning with concept cartoons enhance critical thinking and performance in secondary school economics». Journal of Economic Education Research, 18(1), pp. 1-13. 